\title{
Dinâmica da reprodução e crescimento de Hoplias malabaricus (Bloch, 1794) (Osteichthyes, Erythrinidae) da Represa do Monjolinho, São Carlos/SP.
}

\section{Geraldo Barbieri}

\begin{abstract}
Studies on reproduction dynamics and growth of Hoplias malabaricus were carried out in Monjolinho Reservoir (São Carlos(SP) and the following conclusions were reached to: the reproductive period occurs in September and October. The mean values of replection and gonadosomatic index and condition factor increased during the reproductive period. The size of the first sexual maturation of females was observed at $16,7 \mathrm{~cm}$. The fecundity, that is relatively low, was related with total length and weight of females. The growth curve suggests a slow growth of this species.
\end{abstract}

Hoplias malabaricus, popularmente conhecido como traíra, ocorre em todas as bacias hidrográficas da América do Sul, com exceção da área transandina e dos rios da Patagônia (FOWLER, 1950). É uma espécie bem adaptada a ambientes lênticos, embora possa ser encontrada em rios de pequeno e grande portes. Na fase larval é planctófaga e na adulta essencialmente ictiófaga, com baixa voracidade e com grande resistência à períodos de jejum (PAIVA, 1974). Segundo CARTER \& BEADLE, 1930/32, a traíra, mesmo não apresentando adaptações morfológicas, pode sobreviver em ambientes pouco oxigenados o que explica sua grande capacidade de dispersão e adaptação. Na Represa do Lobo (São Paulo), a espécie apresenta melhores condições alimentares no período que antecede a reprodução sendo que, neste período, a atividade alimentar não é interrompida (BARBIERI et al., 1982).

O presente trabalho tem por objetivo estudar o comportamen to biológico da espécie no que se diz respeito a alguns aspectos da reprodução e crescimento.

\section{MATERIAL E MÉTODOS}

Durante o ano de 1987, 404 exemplares de Hoplias malabaricus (213 fêmeas e 191 machos) foram coletados mensalmente na Represa do Monjolinho (São Carlos/SP). Essa represa apresenta uma área de 4,6 ha com profundidades máximas e média de 3,00 e 1,68 metros, respectivamente. De cada exem- 
plar coletado foram registradas as seguintes variáveis: comprimento total (Lt) em $\mathrm{cm}$, pesos totais em $\mathrm{g}$ do peixe $(\mathrm{Wt})$, da gônada $(\mathrm{Wg})$, do fígado (Wf) e do estômago (Wr). Os estádios de maturação gonadal foram determinados macro e microscopicamente segundo metodologia descrita por BARBIERI \& BARBIERI (1984) para mesma espécie da Represa do Lobo (SP). Foram coletadas 5 a 6 escamas de cada exemplar para identificação e leitura dos anéis etários, as quais foram preparadas segundo metodologia descrita por OOSTEN (1929). Quando os testes estatísticos permitiram, os dados de machos e fêmeas foram analisados conjuntamente.

O período reprodutivo foi estabelecido através da análise da curva de maturação, baseada na variação bimestral do índice gonadossomático medio ( $\overline{\mathrm{I} g})$. Os valores médios do índice hepatossomático (Īhs) e do índice de repleção (Ir) foram lançados em gráfico, em função dos bimestres do ano. Esses índices foram calculados através da expressão peso do orgão/peso do exemplar. 100, após ter sido constatada relação linear pela origem entre as variáveis envolvidas. O fator de condição $(\phi)$ foi estimado através da relação: $\phi=\frac{\mathrm{Wt}}{\mathrm{Lt} \theta}$, onde $\theta$ corresponde ao parâmetro que define o tipo de crescimento da espécie. Os valores médios desse fator $(\phi)$ foram também lançados em função dos bimestres.

O tamanho de $1^{\mathrm{a}}$. maturação gonadal foi considerado como aquele no qual $50 \%$ dos indivíduos da população puderam ser classificados como adultos.

Os ovários destinados à estimativa da fecundidade foram imersos em solução de Gilson modificada (SIMPSON, 1951), permanecendo aí até o desprendimento completo dos ovócitos do tecido conjuntivo e em seguida lavado em álcool $70^{\circ} \mathrm{GL}$. A estimativa do número de ovócitos foi estabelecida em subamostras com auxilio de uma pipeta de Stempell $(2,5 \mathrm{ml})$. A fecundidade foi relacionada com o comprimento e peso das gônadas.

A época de formação dos anéis etários nas escamas foi estabelecida graficamente, através dos comprimentos totais medios bimestrais em cada classe de número de anéis.

A curva de crescimento em comprimento, entendida como a relação entre o comprimento total e a idade, foi obtida com base na expressão matemática de von BERTALANFFY (1938), após ter sido verificada sua validade através da transformação Ford-Walford (WALFORD, 1946).

A curva de crescimento em peso total, entendida como a relação entre o peso total e a idade, foi obtida através do método dedutivo, a partir das expressões matemáticas da curva de crescimento em comprimento e da relação peso total/comprimento total do peixe.

As metodologias básicas para estimativas dos parâmetros matemáticos das expressões encontram-se em BEVERTON \& HOLT (1957), RICKER (1971), SANTOS (1978) e VAZZOLER (1981).

\section{RESULTADOS E DISCUSSÃO}

Como podemos observar através da análise da curva de maturação, a atividade reprodutiva da espécie é mais acentuada nos meses de setembro e outubro 
quando os valores do índice gonadossomático são maiores. $\mathrm{O}$ índice gonadossomático médio (Fig. 1A) mostra-se baixo no período reprodutivo em relação a peixes de desova total, sugerindo tratar-se de espécie de desova parcelada, fato observado para a mesma espécie, por CARAMASCHI et al. (1982) e BARBIERI \& BARBIERI (1984). Outra observação que confirma o tipo de desova da traíra é a presença de alta freqüência de indivíduos classificados como semi-desovados, característica de peixe com desenvolvimento assincrônico dos ovócitos e portanto de desova parcelada. Segundo NIKOLSKY (1963 e 1969) o parcelamento da desova, característica de peixes tropicais, é considerado como uma adaptação da espécie para evitar a competição pelo local de desova e alimento para as larvas.

Apesar do período de desova da traíra ser de apenas dois meses, esse comportamento pode ser considerado como uma estratégia de manutenção da espécie.

BARBIERI et al. (1982) verificaram comportamento semelhante para a espécie da Represa do Lobo (SP). Entretanto, I HERING (1938) e PAIVA (1979) citam como época de desova dessa espécie os meses de outubro-novembro, e primeiro e segundo trimestres do ano, respectivamente, no nordeste brasileiro. AZEVEDO \& GOMES (1943) e CARAMASCHI (1979) verificaram que H. malabaricus inicia a desova em novembro e se estende até fevereiro em Pirassununga/SP e até março na Represa do rio Pardo (Botucatu/SP).

Os valores medios do índice gonadossomático observados para os exemplares machos da Represa do Monjolinho foram baix os seguindo o padrão observado para a maioria dos teleósteos.

A variação do índice de repleção (Fig. 1B) sugere que essa espécie apresenta melhores condições alimentares no período que antecede a reprodução e durante a reprodução. O mesmo comportamento foi observado por BARBIERI et al. (1982) para a espécie da Represa do Lobo (SP). Os resultados obtidos por PAIVA (1974) para a espécie do nordeste brasileiro estão de acordo com nossas observações quanto à continuidade da atividade alimentar no período reprodutivo.

Durante o período de maior atividade reprodutiva, verificamos maiores valores médios do índice hepatossomático nas fêmeas (Fig. 1C), sugerindo maior síntese de proteínas nesse período, fato não observado nos machos.

O fator de condição $(\phi)$ pode ser tomado como uma medida do "bem estar" geral do peixe. Entretanto, alguns fatores como desenvolvimento gonadal, grau de repleção do estômago, etc, podem influenciar esse fator. Os resultados obtidos no presente trabalho (Fig. 1D), sugerem que o fator de condição pode ser considerado como bom indicador do período reprodutivo da espécie. O mesmo comportamento foi observado para a população de $H$. malabaricus do Reservatório de Itaipú (Núcleo de Pesquisa . . . 1987).

O tamanho de primeira maturação (Fig. 2) foi estimado em $16,7 \mathrm{~cm}$ de comprimento total, correspondendo ao 20. ano de vida (Fig. 4B).

A partir de $23 \mathrm{~cm}$ de comprimento total, 100\% das fêmeas estão aptas à reprodução. Segundo CARAMASCHI (1979) a traíra da Represa do Rio Pardo atingiu a primeira maturação ao redor de $13,5 \mathrm{~cm}$ de comprimento total e segundo esta autora alguns fatores como fotoperíodo e temperatura alta podem favorecer a precocidade sexual da espécie, o que explicaria citação de LOWE- 

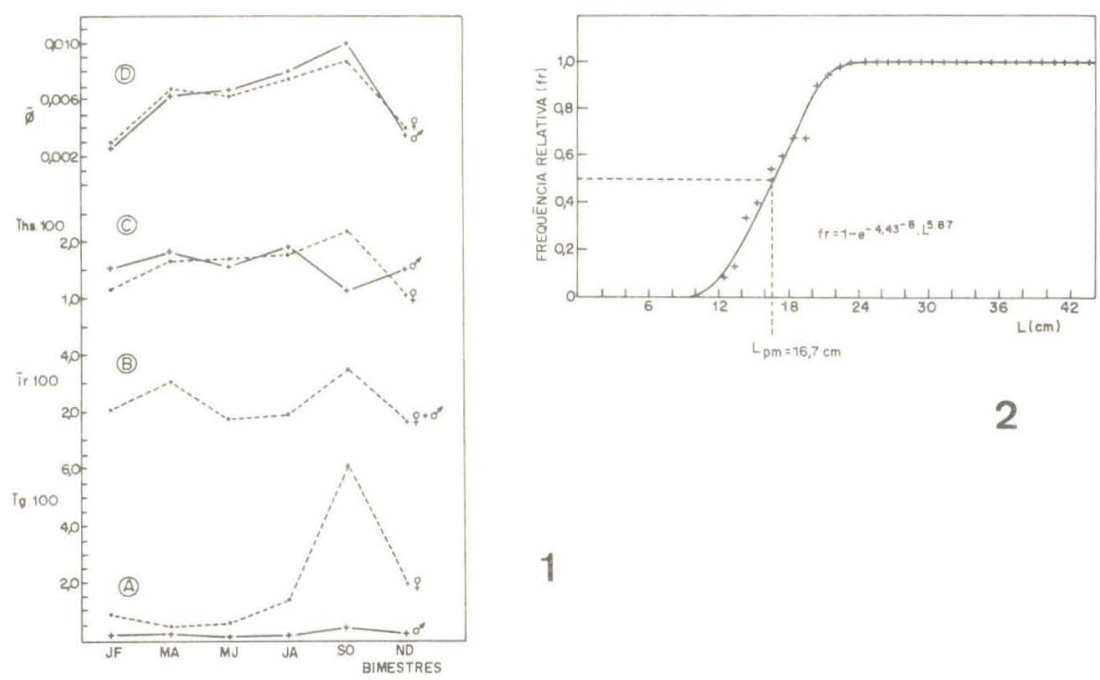

FIG. 1 - Variação média bimestral do índice gonadossomático (A), do índice de repleção (B), do índice hepatossomático (C) e do fator de condição (D).

FIG. 2 - Frequêencia relativa de fêmeas adultas por classe de comprimento totais $(\mathrm{Lpm}=$ tamanho de primeira maturação gonadal).

McCONNELL (1964) para as traíras da savana Rupunini (Guianas), com tamanho de primeira maturação estimado em $20 \mathrm{~cm}$ de comprimento padrão.

A estimativa da fecundidade em peixes de desova parcelada é mais difícil devido à dificuldade de se distinguir os ovócitos de reserva daqueles em desenvolvimento e de se prever as taxas de reabsorção (BAGENAL, 1978). Assim, é conveniente estimar o total de ovócitos vitelinados (maduros e em maturação) a serem postos durante o período reprodutivo. A fecundidade média da população em estudo foi estimada em 7875 ovócitos. Essa estimativa pode ser considerada baixa quando comparada à outros teleósteos de água doce. Segundo NIKOLSKY (1963) são mais fecundas as espécies que não protegem a prole e que realizam desovas múltiplas ou que não dispõem de mecanismos eficientes de defesa. A traíra possui desovas múltiplas e parece não dispensar cuidados especiais às suas posturas.

Essa relação inversa entre a fecundidade e os cuidados parentais com a prole, relatada por LAGLER et al. (1977) não foi constatada para $H$. malabaricus da Represa do Monjolinho. Acreditamos que, nesse caso, a fecundidade está mais relacionada ao baixo suprimento alimentar e à grande densidade populacional daquele ambiente lêntico. Esta observação está de acordo com os relatos de BAGENAL (1966) para outras espécies de peixes.

A fecundidade de $H$. malabaricus da Represa do Monjolinho foi associada ao comprimento total (Fig. 3A) e peso total (Fig. 3B). Foi constatado que o número de ovócitos aumenta em função destas variáveis, sendo relação potencial para comprimento e linear para o peso, comportamento semelhante ao observado por CARAMASCHI (1979) para as traíras da Represa do Rio Pardo (SP). 

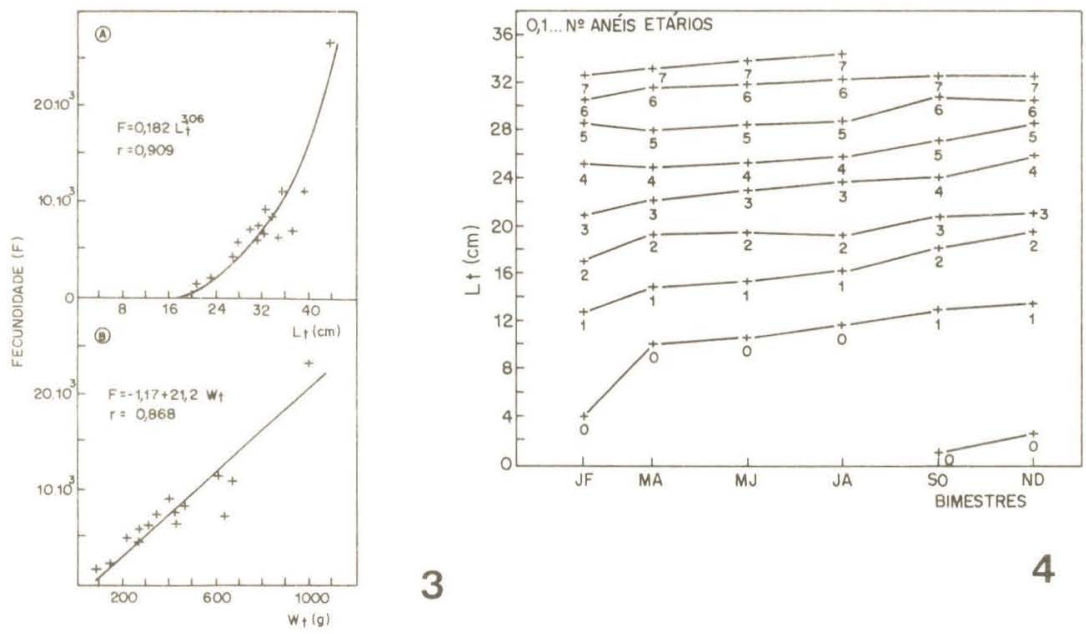

3

\section{4}

FIG. 3 - Relação fecundidade/comprimento total (A), relação fecundidade/peso total (B) das fêmeas.

FIG. 4 - Variação bimestral dos comprimentos médios em função do número de anéis nas escamas.

Outro aspecto que também tem gerado controvérsias nas análises populacionais de peixes tropicais com desova parcelada, é a validade do método da leitura de anéis etários em escamas. Alguns autores (PANNELLA, 1974; NEKRASOV, 1979), mesmo reconhecendo haver certa dificuldade na distinção de anéis falsos e verdadeiros, têm defendido a validade dessa metodologia e consideram o processo reprodutivo como fator responsável pela sua formação. As escamas de traíras da Represa do Monjolinho apresentam falsos anéis, considerados por PAIVA (1974) como anéis duplos e que foram identificados e não computados nas análises. Foram observados até 7 anéis nas escamas e não houve diferença quanto ao sexo. Os comprimentos médios de indivíduos com mesmo número de anéis, foram lançados em gráfico, em função dos bimestres (Fig. 4). Como podemos observar, os anéis são formados nos meses de setembrooutubro, sugerindo que os mesmos são decorrentes de um retardamento no crescimento em comprimento no período reprodutivo. Nesse mesmo período, surgem na população, indivíduos jovens que irão constituir nova classe etária e que possuem em média 37 dias de idade.

Através dos pontos empíricos a metodologia de WALFORD (1946) foi aplicada (Fig. 5A), confirmando a validade da expressão de BERTALANFFY (1938) para o ajustamento da curva de crescimento em comprimento (Fig. 5B). A relação peso/comprimento (Fig. 5C) segue o mesmo padrão dos demais teleósteos. Com as expressões matemáticas dessas duas relações, a curva de crescimento em peso foi obtida (Fig. 5D). A boa aderência dos pontos empíricos à curva teórica, confirma a validade de metodologia empregada. 


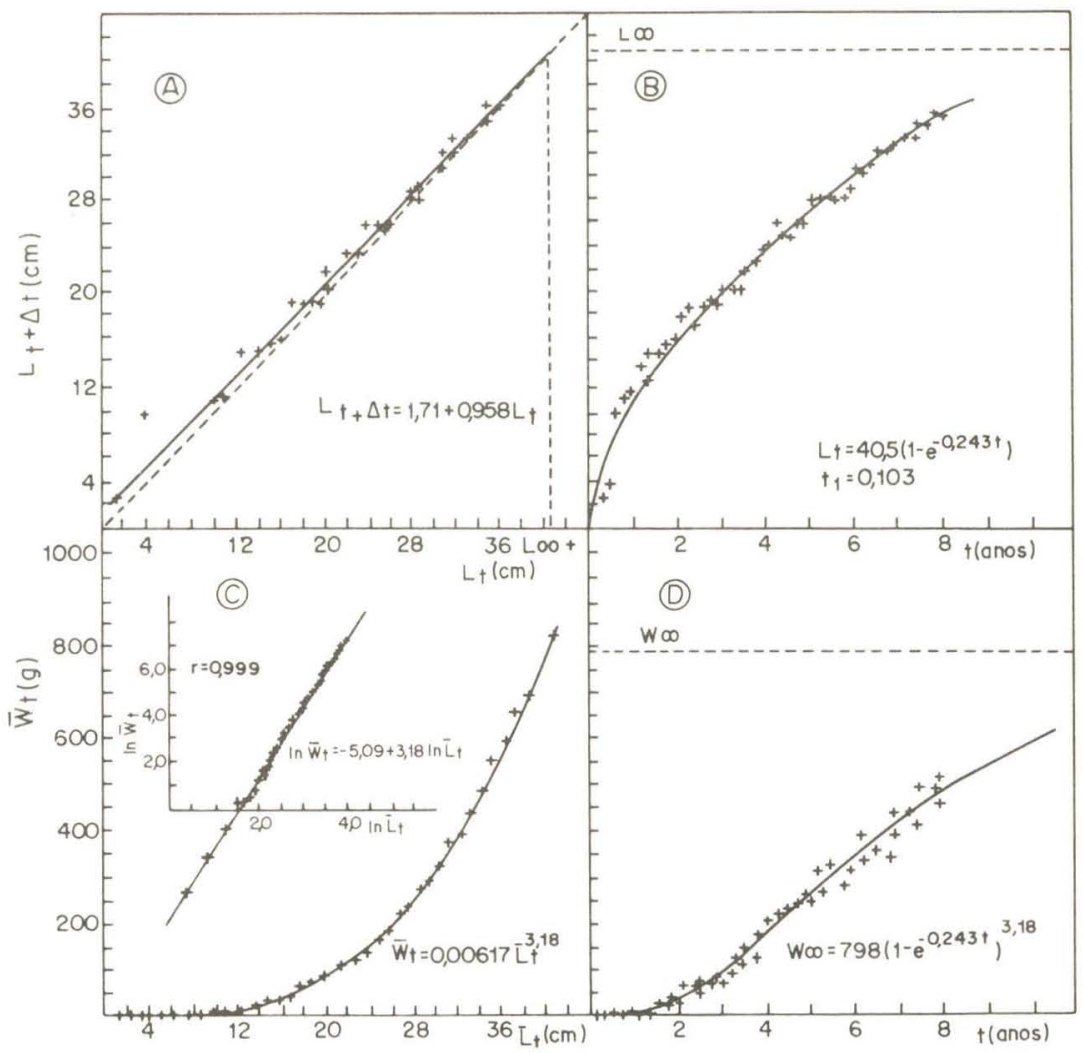

FIG.5 - Transformação Ford-Walford (A), curva de crescimento em comprimento, ajustada (B), relação peso total médio/comprimento total médio (C) e curva de crescimento em peso (D).

A espécie apresenta crescimento lento $(\mathrm{K}=0,243)$ e isométrico $(\theta=3,18)$. De acordo com GRIMES (1978), valores baixos na taxa de crescimento são características de especie cuja alimentação ocorre em niveis tróficos mais elevados. O crescimento da espécie em estudo segue o mesmo padrão da maioria dos teleósteos, onde os incrementos em comprimento são maiores nos indivíduos jovens, ocorrendo o inverso com relação ao incremento em peso. $\mathrm{O}$ crescimento de $H$. malabaricus é mais rápido nos dois primeiros anos, antes de atingir o tamanho de primeira maturação gonadal. Nesta idade atingem cerca de $40 \%$ do comprimento assintótico $\left(\mathrm{L}_{\infty}=40,5 \mathrm{~cm}\right)$. Segundo LAGLER et al. (1977) essa diminuição da velocidade de crescimento é decorrente da demanda de nutrientes para a formação dos gametas nos indivíduos adultos e dos gastos energéticos no processo da desova. 
No presente estudo, verificamos a ocorrência de uma considerável variação no comprimento entre indivíduos com mesmo número de anéis nas escamas. PAPAGEORGIOU (1979) sugere que essa variação pode ser devida à competição pelo alimento ou inerente à taxa de crescimento individual. Segundo AGOSTINHO (1985) o período prolongado de desova pode levar a esta variação. Assim, peixes que eclodem primeiro podem alcançar tamanhos consideráveis quando as últimas posturas estão em curso. Considerando que os maiores são competidores mais eficientes e aptos a conseguirem mais alimentos, podem, eventualmente, alcançar maiores tamanhos.

\section{CONCLUSÕES}

De acordo com os objetivos propostos, podemos concluir para a espécie Hoplias malabaricus da Represa do Monjolinho (São Carlos/SP):

1. o período reprodutivo está compreendido entre os meses de setembro e outubro.

2. apresenta melhores condições alimentares no período que antecede a reprodução sem contudo diminuir atividade nesse período. Os valores do índice hepatossomático diminuem logo após a reprodução.

3. o fator de condição pode ser considerado como um bom indicador do período de desova.

4. o tamanho de primeira maturação gonadal foi estimado em $16,7 \mathrm{~cm}$, correspondendo ao $2^{\circ}$. ano de vida das fêmeas. A partir de $23 \mathrm{~cm}$ de comprimento total todas as fêmeas estão aptas à reprodução.

5. a fecundidade média foi estimada em 7875 ovócitos, considerada baixa em relação à maioria dos teleósteos. A fecundidade quando associada ao comprimento e peso das fêmeas, apresenta relação potencial e linear, respectivamente.

6. o crescimento é lento e do tipo isométrico. A formação dos anéis ocorre por período reprodu tivo.

\section{REFERÊNCIAS}

AGOSTINHO, A. A., 1985. Estrutura da população, idade, crescimento e reprodução de Rhinelepis aspera (Agassiz, 1829) (Osteichthyes, Loricariidae) do Rio Paranapanema, PR, São Carlos, 231 p. Tese de Doutoramento. Universidade Federal de São Carlos.

AZEVEDO, P. de \& A. L. GOMES, 1943. Contribuição ao estudo da biologia da traíra Hoplias malabaricus (Bloch, 1794). Bolm Industr. Anim., São Paulo, 5(4): 15-64.

BAGENAL, T. B., 1966. The ecological and geographical aspects of fecundity of the plaice. J. mar. biol. Ass. U. K. , 46(1):161-186. 
BAGENAL, T. B., 1978. Aspects of fish fecundity. In GERKING, S. D. Ecology of freshwater fish production, Blackwell Scientific Publications, Oxford, p. 75-101.

BARBIERI, G., J. R. VERANI \& M.C.BARBIERI, 1982. Dinâmica quantitativa da nutrição de Hoplias malabaricus (Bloch, 1794) (Pisces, Erythrinidae), na Represa do Lobo (Brotas-Itirapina/SP). Revta bras. Biol., Rio de Janeiro, 42(2):295-302.

BARBIERI, M. C. \& G. BARBIERI, 1984. Observations on the morphology and histology of the testes of Hoplias malabaricus (Bloch, 1794) (Pisces, Erythrinidae) from the Lobo Reservoir (Brotas/Itirapina-SP). An. Sem. Reg. Ecol., IV :203-215.

BERTALANFFY, L. von, 1938. A quantidade theory of organic growth. Human Biology, $10(2): 181-213$

BEVERTON, R. J. \& S. J. HOLT, 1957. On the dynamics of exploited fish populations. Min. Agric. Fish and Food. Fish. Invest., 2 (19), 533 p.

CARAMASCHI, E. M. P., 1979. Reprodução e alimentação de Hoplias malabaricus (Bloch, 1794) na Represa do Rio Pardo (Botucatu, SP) (Osteichthyes, Cypriniformes, Erythrinidae). São Carlos, 144 pp. Dissertação de Mestrado. Universidade Federal de São Carlos.

FOWLER, H. W., 1970. Os peixes de água doce do Brasil. Archos Zool. Est. S. Paulo, 6: $362-364$.

GRIMES, C. B., 1978. Age, growth and length-weight relationship of vermilion snapper, Rhomboplites aurorubens, from north Carolina and South Carolina waters. Trans. Am. Fish. Soc., 107:454-456.

IHERING, R. von, 1938. Biologia da traíra. In AZEVEDO, P. \& A. L. Gomes, Contribuição ao estudo da biologia da traíra, Hoplias malabaricus (Bloch, 1794). Bolm Industr. Anim., São Paulo, 5(4):15-64, 1943.

LAGLER, K. F., J. E. BARDACH, R. R. MILLER \& D. R. PASSINO, 1977. Ichthyology . John Wiley \& Sons, New York, 506 p.

LOWE-McCONNELL, R. H., 1964. The fishes of Rupununi savana district of British Guiana, South America. I. Ecological groupings of fish species and effects of the seasonal cycle on the fish. J. Limn. Soc. (Zool.), 45(304):103-144.

NEKRASOV, V. V., 1979. The causes of annulus formation in tropical fishes. Hidrobiological Journal, 14(2):35-39.

NIKOLSKY, G. V., 1963. The ecology of fishes. Mexico, Academic Press, 352 p.

NÚCLEO DE PESQUISAS EM LIMNOLOGIA, ICTIOLOGIA E AQUICULTURANUPELI A, 1987. Relatório do Projeto "Ictiofauna e Biologia Pesqueira - Reservatório de Itaipú/Fundação Universidade Estadual de Maringá - Paraná,'v. 2, p. 515 -30.

OOSTEN, J., 1929. Life history of the lake herring (Leucichthys ortedi Lesweur), of Lake Huron as revealed by its scale, with a critique on the scale method. Bull. U.S.Fish and Wildlife Service, Fishery Bull., 44:265-448.

PAIVA, M. P., 1974. Crescimento, alimentação e reprodução da traíra, Hoplias malabaricus (Bloch) no Nordeste brasileiro. São Paulo, 32 p. (Tese de Doutoramento, Universidade de Sào Paulo. 
Vol. 6(2), 1989

PANNELLA, G., 1974. Otolith growth patterns: an aid in age determination in temperate and tropical fishes. In BAGENAL, T. B. Ageing of fishes, Unwin Brothers, Surrey, $234 \mathrm{p}$.

PAPAGEORGIOU, N. K., 1979. The length weight relationship, age, growth and reproduction of the roach Rutulus rutulus (L.) in Lake Volvi. J. Fish. Biol., 14(6)529538.

RICKER, W. E. (ed.), 1971. Methods for assessment of fish production in freshwaters, Oxford, Blackwell Scientific Publications, 348 p.

SANTOS, E. P. dos, 1978. Dinâmica de populações aplicada à pesca e piscicultura. São Paulo, Hucitec-Edusp, 129 p.

SIMPSON, A. C., 1951. The fecundity of the plaice. Fish. Inv., 17(5): 1-27.

VAZZOLER, A. E. A. de, 1981. Manual de métodos para estudos biológicos de populações de peixes. Reprodução e crescimento. Brasília. CNPq/PNZ, 108 p.

WALFORD, L. A., 1946. A new graphic method of describing the grow th animals. Biol. Bull. mar. biol. Lab., Woods Hole, 90 (2):141-147. 